\title{
Diyabetik Epiretinal Membranlar Nedeniyle 23 Gauge Pars Plana Vitrektomi Ameliyatı Geçiren Hastaların Klinik Sonuçları
}

\author{
Mehmet Demir ${ }^{1}$, Dilek Güven', Yekta Şendül ${ }^{1}$, Zeynep Acar ${ }^{1}$, Ali Olgun¹
}

\begin{abstract}
ÖZET:
Diyabetik epiretinal membranlar nedeniyle 23 gauge pars plana vitrektomi ameliyatı geçiren hastaların klinik sonuçları

Amaç: Diyabetik epiretinal membranlar nedeniyle 23 gauge pars plana vitrektomi (PPV) ameliyatı geçiren hastaların klinik sonuçlarını sunmak.

Gereç ve Yöntem: Diyabetik epiretinal membranların sebep olduğu görme azlığı, maküla ödemi (MÖ) ve/ veya metamorfopsi nedeniyle sadece PPV veya PPV ile birlikte fakoemülsifikasyon ve göz içi lens (GiL) ameliyatı geçiren hastaların ameliyat öncesi ve sonrası en iyi düzeltilmiş görme keskinliği (EiDGK), göz içi basınçları (GiB), merkezi maküla kalınlıkları (MMK) geriye dönük olarak incelendikten sonra karşılaştırıldı ve gelişen komplikasyonlar açısından incelendi.

Çalışmaya ameliyattan önce panretinal fotokoagülasyon tedavisi görmüş, psödofakik ya da görmeyi etkileyen lens kesafeti olmayan ancak ileride vitrektomi sekeli olarak gelişecek katarakt nedeniyle kombine cerrahi geçiren hastalar alındı.

Bulgular: Yirmi dokuz hastanın (17 kadın, 12 erkek) 30 gözü incelendi. Yaş ortalaması $61.8 \pm 8.2$ yıl, takip süresi $13 \pm 7.9$ ay idi. Ameliyat öncesi ortalama EiDGK $0.65 \pm 0.25$ logMAR; ameliyat sonrası ortalama EiDGK $0.54 \pm 0.29$ logMAR idi $(p=0.29)$. MMK ortalaması ameliyat öncesi $474 \pm 81 \mu \mathrm{m}$ ve ameliyat sonrası $295 \pm 52$ $\mu \mathrm{m}$ bulundu $(\mathrm{p}<0.001)$.

EiDGK, on üç gözde (\%43.4) arttı, 10 gözde (\%33.3) değişmedi ve 7 gözde (\%23.3) azaldı. Posterior kapsül opasifikasyonu (PCO) 7 olguda kaydedildi. Glokom beş gözde, makülada skar oluşumu iki gözde, lamellar maküler delik üç gözde, tam kat maküler delik bir gözde, rubeosis iridis bir gözde ve optik atrofi iki gözde, gelişti.

Sonuç: EIDGK, retina önünde diyabetik membran gelişmiş panretinal lazer fotokoagülasyon yapılmış diyabetik retinopatli gözlerde membran soyma ameliyatından sonra gözlerin \%43.4'de artış, \%33.3'de ise değişiklik göstermedi. Ortalama MMK'da anlamlı incelme izlendi. Glokom, maküla ile ilgili yan etkiler ve arka kapsül opasifikasyonu ameliyat sonrası en sık izlenen komplikasyonlardı.
\end{abstract}

Anahtar kelimeler: Diyabetik epiretinal membran, fakoemülsifikasyon, 23 gauge vitrektomi

ABSTRACT:

Clinical Outcomes of 23-gauge pars plana vitrectomy for diabetic epiretinal membranes in diabetic patients

Objective: To report the clinical outcomes of 23-gauge (G) pars plana vitrectomy (PPV) for epiretinal membranes (ERM) in diabetic patients.

Materials and Methods: This retrospective study included 30 eyes of 29 diabetic patients who underwent 23-G PPV only or combined with phacoemulsification and intraocular lens (IOL) implantation for ERM. All patients complained low visual acuity and/or metamorphopsia secondary to ERM with or without macular edema (ME). Phacoemulsification was performed for the lack of re-operation for cataract that will develop after vitrectomy. Preoperative and postoperative values of best corrected visual acuity (BCVA), central macular thickness (CMT), intraocular pressure (IOP) and complications of surgery were analyzed.

Results: Mean age was $61.8 \pm 8.2$ years old and follow up time was $13 \pm 7.9$ months. Preoperative mean BCVA was $0.65 \pm 0.25$ logarithm of the minimum angle of resolution (logMAR) and postoperative mean BCVA was $0.54 \pm 0.29 \log M A R(p=0.29)$. Preoperative mean CMT was $474 \pm 81 \mu \mathrm{m}$ and postoperative mean CMT was $295 \pm 52 \mu \mathrm{m}(p<0.001)$.

Postoperatively, in 13 eyes (43.4\%), BCVA was increased. In 10 eyes (33.3\%), BCVA was not changed and in 7 eyes (23.3\%), BCVA was decreased at the end of follow-up. Posterior capsul opacification was recorded in 7 eyes. Glaucoma in five, macular scar formation in two, lamellar macular hole formation in three, full-thickness macular hole formation in one, rubeosis iridis in one and optic atrophy in two eyes were recorded.

Conclusions: BCVA was increased in $43.4 \%$ and not changed in $33.3 \%$ eyes after PPV for diabetic ERM. Mean CMT was significantly decreased after PPV. Postoperatively glaucoma, macular side events, and posterior capsular opacification were the most frequent complications.

Key words: Diabetic epiretinal membrane, phacoemulsification, 23 gauge vitrectomy

Ş.E.E.A.H. Tıp Bülteni 2014;48(4):282-6
'Şişli Hamidiye Etfal Eğitim ve Araştırma Hastanesi, Göz Kliniği, İstanbul - Türkiye

Yazışma Adresi / Address reprint requests to: Mehmet Demir,

Halaskargazi Caddesi, Etfal Sokak,

Şişli Hamidiye Etfal Eğitim ve Araştırma

Hastanesi, Göz Kliniği, Şişli, İstanbul - Türkiye

E-posta / E-mail:

drmehmetfe@hotmail.com

Geliş tarihi / Date of receipt: 16 Mayıs 2014 / May 16, 2014

Kabul tarihi / Date of acceptance: 24 Haziran 2014 / June 24, 2014 


\section{GíRiş}

Diyabetik retinopati, gelişmiş toplumlarda en sık görülen görme kaybı sebebidir. Pars plana vitrektomi (PPV) diyabetik hastalarda sıkça yapılan göz ameliyatlarından biridir. Pars plana vitrektomiden sonra katarakt gelişimi sıkça görülen bir komplikasyondur. Vitrektomi ameliyatı geçirmiş gözlerde vitreus desteği olmadığı için, katarakt ameliyatı yapılırken komplikasyonlarla karşılaşmak vitrektomi ameliyatı olmamış gözlere göre daha sıktır. Katarakt ameliyatı esnasında arka kapsül yırtılması, lensin vitre içine düşmesi, zonüler lif kaybı, cerrahi esnasında ön kamaranın derinleşmesi sıkça gelişen komplikasyonlar arasındadır (1-3). Bu zorluklar nedeniyle vitrektomi ameliyatı yapılırken, fakoemülsifikasyon ameliyatı özellikle akomodasyon ihtiyacı olmayan hastalarda vitrektomi ile beraber sıkça yapılmaktadır. Yirmi üç gauge (G) PPV diyabetik epiretinal membranlar için etkili ve güvenli bir yöntemdir $(4,5)$.

Bu çalışmada panretinal fotokoagülasyon yapılmış diyabetik retinopatiye bağlı epiretinal membranlar (ERM) nedeniyle 23 G PPV ameliyatı geçiren hastaların klinik sonuçlarını sunmayı amaçladık.

\section{GEREÇ VE YÖNTEM}

Maküla ödemi ve/veya metamorfopsiye neden olan diyabetik ERM nedeniyle 23 gauge PPV geçiren psödofakikvePPVilebirliktefakoemülsifikasyon+Gil ameliyatı geçiren fakik hastalar geriye dönük olarak incelendi. Bütün hastalar proliferatif diyabetik retinopati nedeniyle ameliyattan önce panretinal lazer fotokoagülasyon tedavisi görmüşlerdi. Hastalar yaş, cinsiyet, ameliyat öncesi ve sonrası en iyi düzeltilmiş görme keskinliği (EiDGK, logarithm of the minimum angle of resolution (logMAR), merkezi maküla kalınlığı (MMK), göz içi basınç (GiB) değerleri ile ameliyat sonrası gelişen komplikasyonlar açısından incelendi.

Ameliyat öncesi makülada tam kat ya da lamellar delik olanlar, göz tembelliği, yaşa bağlı maküla dejeneresansı, glokomu, travma sonrası makülopatisi, birden fazla vitrektomi ameliyatı geçirenler, ameliyat öncesinde görme keskinliğini düşürecek düzeyde lens kesafeti, intraoküler hemorajisi ve takip süresi dört aydan kısa olanlar çalışma dışı bırakıldı.

Pars plana vitrektomi limbusun $3.5 \mathrm{~mm}$ dışında pars plana'dan $23 \mathrm{G}$ trokar sistemi kullanılarak yapıldı. Katarakt ameliyatına başlamadan önce 3'lü trokarlar yerleştirildikten sonra tıpa ile kapatıldı. Fakoemülsifikasyon cerrahisi süperior korneal alanda 3 mm genişliğindeki tünel kesiden yapıldı. Ön kamara viskoelastik madde ile doldurulduktan sonra, 5-6 mm çapında kapsüloreksis yapıldı. Hidrodiseksiyonu takiben fakoemülsifikasyon ve korteks temizliği gerçekleştirildi. Ön kamara ve kapsüler boşluğa \%1.0 sodyum hyalüronat verildi, katlanabilir Gí kapsüler boşluğa yerleştirildi ve viskoelastik madde aspire edildi, ana korneal giriş yerine bir adet 10/0 naylon sütür konulduktan sonra arka segment cerrahisine geçildi. Standart 3 portlu pars plana vitrektomi nonkontakt geniş açılı görüntüleme sistemi (Eibos SPXL, Möller-Wedel, Almanya) kullanılarak gerçekleştirildi. Vitrektomi esnasında, kor vitrektomi, posterior hyaloid membran ve epiretinal membran soyulması, periferik vitreus temizliği ve sıvı- sülfürhekzaflorid (SF6) değişimi yapıldı. Vitreus görünürlülüğünü artırmak için triamsinolon asetat kullanıldı. Diyabetik epiretinal membranların boyanmasında dual boya kullanıldı. Damarsal ark içindeki membranlar temizlendi. Membran soyma işlemleri esnasında vitrektomi probu veya pik ile membranı kaldırma işlemleri yapıldı. Yatay ve dikey vitrektomi makasları sıkı membran- retina bağlantılarını ayırma işlemelerinde kullanıldı.

Gaza bağlı gelişebilecek postoperatif komplikasyonları önlemek amacıyla, işlemin sonucunda pupil kontraksiyonu için miyotik olarak karbakol kullanıldı. Postoperatif olarak 24 göze \%1 tropikamid $4 \times 1$ / gün, 5x1/gün topikal kortikosteroid ve $5 \times 1$ /gün topikal antibiyotik kombinasyonu 4 hafta boyunca uygulandı. Altı gözde görülen yoğun fibrinöz ön kamara reaksiyonu için \%0.1 topikal deksametazon $(12 \times 1 /$ gün 2 hafta ve $8 \times 1 /$ gün 2 hafta) kullanıldı. Bu çalışma etik kurul tarafından onaylandı ve Helsinki deklerasyonuna uygun olarak gerçekleştirildi. Tüm hastaların imzalı onamları alındı. Preoperatif ve postoperatif veriler eşleştirilmiş t testi kullanılarak karşılaştırıldı. P değerinin <0.05 olması istatistiksel açıdan anlamlı kabul edildi. 


\section{BULGULAR}

Yirmi dokuz (17 kadın, 12 erkek, ortalama yaş: $61.8 \pm 8.2$ yıl) hastanın 30 (14 psödofakik, 16 fakik) gözü çalışma kapsamına alındı (Tablo 1). Ameliyat sonrası takip süresi $13.0 \pm 7.9$ ay idi. Preoperatif ortalama EIDGK 0.65 $\pm 0.25 \log$ MAR idi. Takip sonrası ortalama EIDGK 0.54 $\pm 0.29 \log$ MAR idi. Postoperatif olarak 13 gözde (\%43.4) görme keskinliği arttı, 10 gözde (\%33.3) görme aynı düzeyde kaldı ve 7 gözde (\%23.3) görme azaldı. Ameliyat öncesi ortalama MMK $474 \pm 81 \mu \mathrm{m}$ ve ameliyat sonrası ortalama MMK $295 \pm 52 \mu \mathrm{m}$ idi. Ameliyat öncesi ortalama GiB $15.4 \pm 3.7 \mathrm{~mm} \mathrm{Hg}$ ve ameliyat sonrası ortalama GiB 18.0 $\pm 8.2 \mathrm{~mm} \mathrm{Hg}$ idi (Tablo 2). Glokom gelişen 5 olgunun 3'ü kombine cerrahi, iki olgu ise sadece vitrektomi cerrahisi geçirmişti. Ameliyatın bitiminde bütün hastalarda retinal tamponad olarak SF6 kullanılmıştı.

$\mathrm{Bu}$ olgu serisinde ameliyat sonrasında glokom, lamellar veya tam kat maküler hol gelişimi, maküler skar oluşumu, optik atrofi, rubeozis iridis izlendi. Yedi olguda posterior kapsül opasifikasyonu gelişti

Tablo 1: Hastaların demografik özellikleri ve takip süresi

\begin{tabular}{ll}
\hline Parametreler & Değerler \\
\hline Yaş (ortalama $\pm S D)$ yıl & $61.8 \pm 8.2(48-79)$ \\
Cinsiyet $(K / E)$ & $17 / 12$ \\
Takip süresi (ortalama $\pm S . S)$ ay & $13.0 \pm 7.9$ \\
\hline
\end{tabular}

S.D: Standard sapma, K/E: Kadın/Erkek ve bu olgulara YAG lazer kapsülotomi yapıldı. Rubeozis iridis gelişen olguya 1 ay arayla üç kez ön kamaraya bevacizumab enjeksiyonu $2.5 \mathrm{mg} / 0.1 \mathrm{~mL}$ yapıldı. Endoftalmi, intravitreal hemoraji ve retina dekolmanı görülmedi. Hastaların son EIDGK ve MMK ölçümlerinde göz içinde tamponad olanlar değerlendirme dışı bırakıldı.

\section{TARTIŞMA}

Bizim bilgimize göre panretinal fotokoagülasyonu yapılmış epiretinal membranlar nedeniyle görme keskinliğinde azalma, maküla ödemi ve/veya metamorfopsi tarifleyen psödofakik hastalarda PPV, fakik gözlerde ise PPV ile kombine fakoemülsifikasyon cerrahisi ile membranları soyulan diyabetik retinopatili hastalarda klinik sonuçları sunan ilk çalışmadır. Bu çalışmadaki cerrahi için endikasyon ERM'ye bağlı azalmış görme keskinliği veya metamorfopsi idi. Kombine cerrahinin sık görülen ön segment komplikasyonlarından yükselmiş GiB \%4.4-\%23.8 oranlarında bulunmuştur (6-9). Bu sonuçlar bizim çalışmamızla benzerlik gösterir ki bizim çalışmamızda'da kombine cerrahi sonrasında yükselmiş GiB sıklığı \%16.7 bulundu. Yükselmiş GiB'li olgular antiglokomatöz ilaçlarla tedavi edildi. Ön kamaradaki fibrinöz eksudasyon sonrasında gelişen posterior sineşi kombine cerrahiden sonra özellikle de PDR'li olgularda istatistiksel olarak daha yüksek sıklıkta görülür $(10,11)$. Kombine cerrahi sonrası ön segment fibrinöz reaksiyonu \%4.0-\%11.2 olarak

Tablo 2: Hastaların ameliyat öncesi ve ameliyat sonrası verileri

\begin{tabular}{lccc}
\hline Parameterler & Ameliyat öncesi & Ameliyat sonrası & p \\
\hline EiDGK (logMAR) & $0.65 \pm 0.25$ & $0.54 \pm 0.29$ & 0.29 \\
MMK $(\mu \mathrm{m})$ & $474.3 \pm 81.2$ & $295.2 \pm 52$ & $<0.001$ \\
GiB & $15.4 \pm 3.7$ & $18.0 \pm 8.2$ & 0.12 \\
Lamellar M.D & 0 & 3 & 0.083 \\
Tam kat M.D & 0 & 1 & 0.32 \\
Makülada skar oluşumu & 0 & 2 & 0.16 \\
Inatçı MÖ & & 1 & 0.32 \\
Glokom & 0 & 5 & 0.02 \\
Optik atrofi & 0 & 2 & 0.16 \\
Rubeosis iridis & 0 & 1 & 0.23 \\
PS & 0 & 4 & 0.032 \\
PCO & 0 & 7 & 0.08 \\
\hline
\end{tabular}

K/E: Kadın/Erkek, EiDGK: En iyi düzeltilmiş görme keskinliği, GiB: Göz içi basıncı, MMK: Merkezi maküla kalınlığı, PS: Posterior sineşi, PCO: Posterior kapsül opasifikasyonu, MÖ: Maküla ödemi, M.D: Maküler delik 
rapor edilmiştir (7). Bizim çalışmamızda, belirgin postoperatif enflamasyon 6 gözde (\%20) görüldü. Yoğun topikal steroid kullanımı fibrinöz reaksiyonu ortadan kaldırmakla birlikte posterior sineşi sekel olarak 4 olguda tespit edildi. Posterior sineşi kombine prosedürün bir komplikasyonu olarak değerlendirilmiştir ve kombine cerrahi sonrasında posterior sineşi oranını \%4.4-\%9.5 olarak açıklayan araştırmalar mevcuttur $(8,12)$. Bizim çalışmamızda takip süreci sonucunda posterior sineşi oranı \%13.3 olarak saptandı. Neovasküler glokom (NVG), kombine prosedürün önemli bir komplikasyonu olarak belirtilmiştir $(13,14)$. Bu çalışmadaki tüm hastalara, panretinal lazer fotokoagülasyonu uygulanmıştı ve çalışmamızdaki hiçbir olguda NVG gelişmedi. Kombine fakoemülsifikasyon, GiL implantasyonu ve vitrektomi ile daha iyi görsel sonuçlar ve daha düşük sıklıkta postoperatif rubeozis iridis rapor edilmiştir $(7,15)$. Ancak, katarakt cerrahisi vitrektomi ile kombine edildiğinde PDR'si olan hastalarda artmış rubeozis iridis ve NVG riski olduğunu destekleyen görüşler mevcuttur. Bu çalışmada kombine cerahi geçiren bir gözde rubeozis iridis gelişti. Birer ay arayla $3 \mathrm{doz}$ bevacizumab $(2.5 \mathrm{mg} / 0.1 \mathrm{~mL})$ enjeksiyonu ön kamaraya yapıldı, rubeozis iridisin son enjeksiyondan sonraki birinci ay muayenesinde tamamen gerilediği görüldü. Tek başına katarakt cerrahisi ile kıyaslandığında kombine cerrahiden sonra posterior kapsül opasifikasyon (PCO)'nunun daha sık görüldüğünü rapor edilmiştir (16). PPV ve katarakt kombine ameliyatı geçiren olgularda cerrahiden sonra, daha yoğun postoperatif enflamasyon gelişebilir. Bu enflamasyon daha erken ve daha yoğun PCO gelişimine yol açabilir. Bu çalışmada, takip süreci sonunda posterior kapsül opasifikasyon oranı \%23.3 olarak saptandı.

Literatürde idiopatik epiretinal membranı olan ve katarakt ekstraksiyonu yapılan hastalarda görme keskinliğinde belirgin artış ve $M M K^{\prime}$ da belirgin azalma saptanmış (17). Başka bir çalışma, vitrektomi ve katarakt ekstraksiyonu birlikte yapılan hastalarda görme keskinliğinde istatistiksel açıdan anlamIı artış olduğunu rapor etmiştir (18). Katarakt ve intraoküler hemorajisi olan hastalarda yapılan kombi- ne cerrahilerden sonra elde edilen görsel sonuçlar, bizim çalışmamızdaki sonuçlardan daha iyiydi (1923). Bu farkın sebebi olarak bahsi geçen çalışmalara dahil edilen hastaların ameliyat öncesi katarakt ve / veya intraoküler kanama gibi preoperatif görmeyi ciddi anlamda azaltan patolojilerinin olmasına yorumladık. Bizim çalışmada fakoemülsifikasyon cerrahisi PPV'den sonra gelişecek katarakt için gerekli olacak yeni bir operasyonu önlemek amacıyla yapılmıştı. Görmenin azaldığı olgularda bu azalmanın altta yatan hastalık sürecine bağlı olarak gelişen ilerleyici retinal dejenerasyon veya maküler hasara bağlı olduğu düşünüldü. Bu çalışmada görme keskinliğindeki azalmanın majör sebepleri olarak retinopati ile birlikte dirençli maküla ödemi, makülada lamellar veya tam kat delik oluşması, makülada skar dokusu gelişimiydi. Bu çalışmada posterior kapsül opasifikasyonu gelişen gözlerde kapsülotomi yapıldıktan sonraki EIDGK değerleri istatistiksel değerlendirmeye alındı. Bizim çalışmamızda, hastaların tümünde diyabetik retinopati mevcuttu ve tümüne panretinal fotokoagülasyon uygulanmıştı. Yeterli lazer fotokoagülasyonun yapılmış olması ve rubeozis iridis gelişen olguda bevacizumab enjeksiyonu yapılmasının NVG gelişimini önlediğini düşündük.

Bu çalışmanın sahip olduğu kısıtlamalar: kontrol grubu olmaması, geriye yönelik olması, örnek grubunun küçük olması ve hastalarda fundus floresin anjiografi değerlendirmelerinin yapılmamış olmasıydı.

\section{SONUÇLAR}

Bu çalışmada, diyabetik epiretinal membranı olan panretinal lazer fotokoagülasyon yapılmış psödofakik ya da belirgin lens kesafeti olmayan hastalardaki membranları soyma amaçlı vitrektomi cerrahisinden sonra gözlerin \%43.4'de görmede artışa olduğu, glokom, maküler ve PCO patolojilerinin ameliyat sonrasında görülebileceği, panretinal fotokoagülasyon lazer tedavisi ve ön kamaraya bevacizumab enjeksiyonun NVG gelişimini önlediğini izledik. 


\section{KAYNAKLAR}

1. Demetriades AM, Gottsch JD, Thomsen R, Azab A, Stark WJ, Campochiaro PA, et al. Combined phacoemulsification, intraocular lens implantation, and vitrectomy for eyes with coexisting cataract and vitreoretinal pathology. Am J Ophthalmol 2003; 135: 291-6.

2. Lahey JM, Francis RR, Kearney JJ. Combining phacoemulsification with pars plana vitrectomy in patients with proliferative diyabetic retinopathy. A series of 223 cases. Ophthalmology 2003; 110: 1335-9.

3. Honjo M, Ogura Y. Surgical results of pars plana vitrectomy combined with phacoemulsification and intraocular lens implantation for complications of proliferative diyabetic retinopathy. Ophthalmic Surg Lasers 1998; 29: 99-105.

4. Hikichi $T$, Matsumoto N, Ohtsuka H, Higuchi M, Matsushita $T$, Ariga $H$, et al. Comparison of one-year outcomes between 23- and 20-gauge vitrectomy for preretinal membrane. Am J Ophthalmol 2009; 147: 639-43.

5. Sisk RA, Murray TG. Combined phacoemulsification and sutureless 23-gauge pars plana vitrectomy for complex vitreoretinal diseases. Br J Ophthalmol 2010; 94: 1028-32.

6. Ahfat FG, Yuen CHW, Groenewald CP. Phacoemulsification and intraocular lens implantation following pars plana vitrectomy: a prospective study. Eye 2003; 17: 16-20.

7. Pollack A, Landa G, Kleinman G, Katz H, Hauzer D, Bukelman A. Results of combined surgery by phacoemulsification and vitrectomy. Isr Med Assoc J 2004; 6: 143-6.

8. Jun Z, Pavlovic S, Jacobi KW. Results of combined vitreoretinal surgery and phacoemulsification with intraocular lens implantation. Clin Experiment Ophthalmol 2001; 29: 307-11.

9. Diolaiuti S, Senn P, Schmid MK, Job O, Maloca P, Schipper I. Combined pars plana vitrectomy and phacoemulsification with intraocular lens implantation in severe proliferative diyabetic retinopathy. Ophthalmic Surg Lasers Imaging 2006; 37: 468-74.

10. Treumer F, Bunse A, Rudolf M, Roider J. Pars plana vitrectomy, phacoemulsification and intraocular lens implantation. Comparison of clinical complications in a combined versus twostep surgical approach. Graefes Arch Clin Exp Ophthalmol 2006; 244: 808-15.

11. Pinarci EY, Bayar SA, Sizmaz S, Yesilirmak N, Akkoyun I, Yilmaz G. Anterior segment complications after phacovitrectomy in diyabetic and nondiyabetic patients. Eur J Ophthalmol 2013; 23: 223-9.

12. Lahey JM, Francis RR, Fong DS, Kearney JJ, Tanaka S. Combing phacoemulsification with vitrectomy for treatment of macular holes. Br J Ophthalmol 2002; 86: 876-8.
13. Scharwey K, Pavlovic S, Jacobi KW. Combined clear corneal phacoemulsification, vitreoretinal surgery, and intraocular lens implantation. J Cataract Refract Surg 1999; 25: 693-8.

14. Mamalis N, Teske MP, Kreisler KR, Zimmerman PL, Crandall AS, Olson RJ. Phacoemulsification combined with pars plana vitrectomy. Ophthalmic Surg 1991; 22: 194-8.

15. Kadonosono K, Matsumoto S, Uchio E, Sugita M, Akura J, Ohno S. Iris neovascularization after vitrectomy combined with phacoemulsification and intraocular lens implantation for proliferative diyabetic retinopathy. Ophthalmic Surg Lasers 2001; 32: 19-24.

16. Toda J, Kato S, Oshika T, Sugita G. Posterior capsule opacification after combined cataract surgery and vitrectomy. I Cataract Refract Surg 2007; 33: 104-7.

17. Dugas B, Ouled-Moussa R, Lafontaine PO, Guillaubey A, Berrod $J P$, Hubert I, et al. Creuzot-Garcher CP. Idiopathic epiretinal macular membrane and cataract extraction: combined versus consecutive surgery. Am J Ophthalmol 2010; 149: 302-6.

18. Oshima Y, Ohji M, Tano Y. Surgical outcomes of 25-gauge transconjunctival vitrectomy combined with cataract surgery for vitreoretinal diseases. Ann Acad Med Singapore 2006; 35: 175 80.

19. Zheng $Q X, W u R H$, Zhang $Y P, X u M$, Li WS. Anterior segment complications after phacoemulsification combined vitrectomy and foldable intraocular lens implantation. Int J Ophthalmol 2010; 3: 249-54.

20. Morales MC, Araiz J, Herrera I, Castiella G, Corcóstegui I, Corcóstegui G. Clinical outcomes of cataract surgery combined with 23-gauge vitrectomy. Arch Soc Esp Oftalmol 2012; 87: 35362.

21. Wilczyński M, Pikulski Z, Dziegielewski K, Omulecki W. Evaluation of results of combined pars plana vitrectomy and cataract removal. Klin Oczna 2005; 107: 252-5.

22. Wei $Q$, Zhang $Y, L i Y$, Shen $L$. The effect of vitreous surgery combined with intraocular lens implantation in proliferative diyabetic retinopathy. Yan Ke Xue Bao 2004; 20: 88-9.

23. Rivas-Aguiño P, García-Amaris RA, Berrocal MH, Sánchez JG, Rivas A, Arévalo JF. Pars plana vitrectomy, phacoemulsification and intraocular lens implantation for the management of cataract and proliferative diyabetic retinopathy: comparison of a combined versus two-step surgical approach. Arch Soc Esp Oftalmol 2009; 84: 31-8. 